\title{
Promotion of health and physical activity improves the mental health of elderly immigrants: results of a group randomised controlled trial among Turkish immigrants in the Netherlands aged 45 and over
}

\author{
S A Reijneveld, M H Westhoff, M Hopman-Rock
}

J Epidemiol Community Health 2003;57:405-411

See end of article for authors' affiliations

Correspondence to: Dr S A Reiineveld, TNO Prevention and Health, $\mathrm{PO}$ Box 2215, $2301 \mathrm{CE}$ Leiden, Netherlands; SA.Reijneveld@pg.tno.nl

Accepted for publication 6 November 2002

\begin{abstract}
Objectives: Older immigrants from non-industrialised countries are a growing group, they have comparatively many health problems and are often hard to reach through health promotion and other preventive services. The aim of this study was to assess the effect of a short health education and physical exercise programme on the health and the physical activity of Turkish first generation elderly immigrants.

Design: Randomised controlled trial.

Setting: Welfare services in six Dutch cities.

Participants: 126 people born in Turkey and aged 45 years and over, of whom 92 completed the trial.

Intervention: Eight, two hour sessions consisting of health education and exercises. Topics in health education focused on means to maintain a good health. Education was adapted to the culture and knowledge of older Turks and offered by a Turkish peer educator, in Turkish.

Main outcome measures: Physical and mental wellbeing, and mental health based on the SF-12/36; knowledge on health and disease; physical activity.

Results: Participants were highly disadvantaged; $52 \%$ had not completed primary school and $49 \%$ had considerable problems in speaking Dutch. Participants in the intervention group showed an improvement in mental health (effect size: 0.38 SD (95\% confidence intervals 0.03 to 0.73$), p=0.03$ ); the oldest subgroup also in mental wellbeing (effect size 0.75 SD (0.22 to 1.28), $p=0.01$ ). No improvements were seen in physical wellbeing and activity, nor in knowledge.

Conclusions: Health education and physical exercise improve the mental state of deprived immigrants. Painstaking cultural adaptations to contents and method of delivery are essential to reach this effect.
\end{abstract}

$\mathrm{H}$ ealth problems among older immigrants from nonindustrialised countries are a rapidly increasing challenge for public health in most countries in Western Europe. Not only is the number of immigrants increasing because of political and economic development, ${ }^{1}$ but rates of health problems, such as cardiovascular, musculoskeletal, and mental health problems, among these immigrants are also comparatively high. ${ }^{1-10}$ The resulting public health challenge can be expected to grow in future as many of these immigrant groups are comparatively young, showing the need to promote the health of these groups.

Cultural differences and practical problems such as language barriers and illiteracy make it rather difficult to reach these groups through health promotion and other preventive services. ${ }^{79}{ }^{10}$ Several examples exist of specific programmes to contact these hard to reach groups. ${ }^{11-13}$ Formal evidence on the effectiveness of such programmes is lacking, however.

Turks are the biggest group of immigrants from nonindustrialised countries in the Netherlands. They were recruited by Dutch employers in the 1970s for unskilled work. ${ }^{214}$ In 2001, 2\% of the Dutch population (320 000 people) was of Turkish descent (that is, at least one parent born in Turkey); 14\% was aged 45 and over. The 45 and over age group had almost all been born in Turkey (http://www.cbs.nl/en/ statline/, assessed 30 April 2002). Two studies are available that comprised Turkish immigrants and indigenous Dutch, ${ }^{2516}$ with results of the first one reported for those aged
$16-64,{ }^{2}$ and results of the second one reported in two publications for those aged $55-74^{15}$ and $35-74{ }^{16}$ respectively. The results of these studies show that Turkish immigrants have a lower educational and income level, ${ }^{2}{ }^{15}$ and report a poorer health, ${ }^{2}{ }^{15}$ more health complaints, ${ }^{2}$ more chronic physical limitations, ${ }^{2}$ and a lower physical activity, ${ }^{16}$ compared with indigenous Dutch of the same age. Differences are especially large regarding the prevalence of long term physical limitations (odds ratio for Turks compared with indigenous Dutch: 13.1 (95\% confidence intervals 8.2 to 20.9$)$ ). ${ }^{2}$

We adapted an existing short health promotion programme, "Healthy \& Vital" (formerly known as "Aging Well and Healthily") for the Turkish elderly population. This programme consists of health education and physical exercises. ${ }^{17}{ }^{18}$ It has been shown to increase physical activity, especially in the least active elderly group, to increase knowledge on health and disease, to reduce feelings of loneliness, and to have a number of favourable physiological effects. ${ }^{17}{ }^{18}$ The aim of this study was to assess the effect of the adapted Healthy \& Vital programme on the health and the physical activity of elderly first generation immigrant Turks in the Netherlands, overall and for relevant subgroups.

\section{METHODS}

The study concerned a controlled trial with group randomisation. 


\section{Protocol}

\section{Study population}

The trial took place in the first half of 2001 in welfare services in six cities in the western half of the Netherlands, after approval of the study by the TNO (Netherlands Organisation for Applied Scientific Research) Medical Ethics Committee. Eligible were people born in Turkey aged 45 years and over. Written informed consent was obtained, information was often read to participants as most could not read in any language.

\section{Intervention}

We used the Healthy \& Vital programme but adapted it for Turkish elderly people. The original Healthy \& Vital programme consists of six, two hour sessions to give people aged 65 and over a lead to subsequent health education and physical exercise programmes. ${ }^{17}{ }^{18}$ During the first hour, a peer educator (elderly) provides information on a topic. The topics all relate to maintaining a good health: successful aging, exercise and health, nutrition, physical and mental resistance and endurance, safety in and around the home, and symptoms related to aging. During the second hour, an exercise instructor delivers a low intensity exercise programme, adapted from the US SMILE programme. ${ }^{19}$ This programme consists of the following parts (number of exercises in the programme): warming up (8); exercises for the upper body (10); exercises for the lower body (7); movements for the whole body (5); cooling down upper body (7); and cooling down lower body (4). The exercise sessions are delivered by an (usually aged) exercise instructor, accompanied by tape recorded music, and are graded in terms of difficulty and number of repetitions. The exercises can be performed in sitting, as well as standing position. ${ }^{18}$ Documentation consists of a course book for participants, comprehensive manuals for the peer educator, the exercise instructor, and the local organiser of the programme, as well as a video tape for the exercise instructor explaining all the exercises.

The Healthy \& Vital programme was adapted in three steps to suit Turkish elderly people. The first step was determining the feasibility of an adaptation based on three focus group interviews. All participants in these interviews $(n=12)$ had long experience in providing health and welfare information to Turks. Results showed that a combination of health education and physical exercise would work best. The health education should be adapted to the Turkish culture, however, and be extended by general medical knowledge and specific complaints (because of low levels of prior knowledge). For instance, examples regarding nutrition should be based on a traditional Turkish diet, examples regarding safety should exclude cycling as many Turkish immigrants do not cycle, and basic information on human anatomy and physiology and on some health complaints (back problems in men and menopause problems in women) should be added. The interviews further confirmed that the level of physical activity of Turkish immigrants was mostly lower than that of the average Dutch person, ${ }^{16}$ and that they had physical limitations at an earlier age. ${ }^{2}$ Therefore the exercise component was extended to eight weeks and recruitment was widened to include younger people ( 45 years and over). The cultural background of the target group prohibited a mixing of genders in the groups, and supported the inclusion of those aged 45 and over ("being old means having grandchildren"). Finally, because of language difficulties and cultural differences, the health education had to be delivered in Turkish, preferably by a Turkish peer educator. ${ }^{20}$

In a second step, two health educators adapted and extended the education part, based on their long experience with the target group and on the available literature. They entirely rewrote the manual for the peer educator and added two sessions: one on the human body and one on gender specific complaints of older people (covering different topics for men and women). Furthermore, they removed almost all written text for participants and replaced this text by figures covering the main topic of each session, supported by some one line captions in Turkish. The exercise part was entirely examined by five exercise instructors with long experience in working with adult and elderly Turks. This examination showed that only comparatively minor modifications were needed, for instance the kind of accompanying music during exercises should changed, exercises should be shown instead of verbally explained, and absence of persons of the other gender during exercising should be guaranteed. Furthermore, a separate instructor manual was needed that focused on working with older immigrants. This was written by a physiotherapist with a long experience in physical exercise (MHW), supported by the aforementioned exercise instructors.

In a third step, the entire programme was piloted in a group of 12 Turkish women. Each of the eight sessions was evaluated, leading to minor modifications in the manuals and materials. For instance, the way in which quantities regarding nutrition were recommended had to be changed. This pilot also resulted in a revision of the manual for organisers, especially regarding the recruitment of participants and professionals. Recruitment should almost entirely be based on personal contacts, for instance in the mosque or by means of key figures in informal groups, whereas Dutch participants were usually recruited via local papers, etc.

\section{Control condition}

Participants either received the adapted Healthy \& Vital programme or the control programme, the "Ageing in the Netherlands" programme. The second programme consists of six sessions on the available welfare services for the elderly. Five sessions take two hours each, the sixth consists of a half day visit.

\section{Quality of delivery}

To monitor and maximise the quality of delivery of both the intervention and the control programme, organisers, health educators, and exercise instructors received manuals regarding their part of the programme concerned, and a one day instruction on it. During the intervention, local organisers evaluated each session with the health educator and the exercise instructor. In turn, the research team contacted the organisers at least every two weeks regarding problems in delivery of the programme and in attendance. If needed, additional contact occurred with organisers, educators, and instructors. A member of the research group attended at least one meeting of each programme, and could be contacted during the entire programme. Finally, all organisers, health educators, and exercise instructors as well as all participants were interviewed on the quality of delivery of both the intervention and the control programme. They had all been informed at the start of the programmes that such an evaluation would occur.

\section{Measures}

Measures concerned general and physical wellbeing calculated from the Short Form (SF)-12, ${ }^{21}$ mental health calculated from five items of the SF-36 (that is, the MHI-5), ${ }^{22}$ knowledge on health and disease regarding the topics covered (self constructed; 12 items that differed for each interview), and physical activity (shortened Voorrips questionnaire) ${ }^{23}$ Regarding knowledge on health and disease we first constructed an item bank of questions concerning the topic of each session. These questions were discussed in the research team; those approved were randomly assigned to either the first or the second questionnaire. Regarding the Voorrips questionnaire, after discussion in the project team and with those involved in the pilot (at step 3), we excluded the items on sports activities as these mostly concerned activities that Turkish elderly people, especially the women, would never undertake. A 


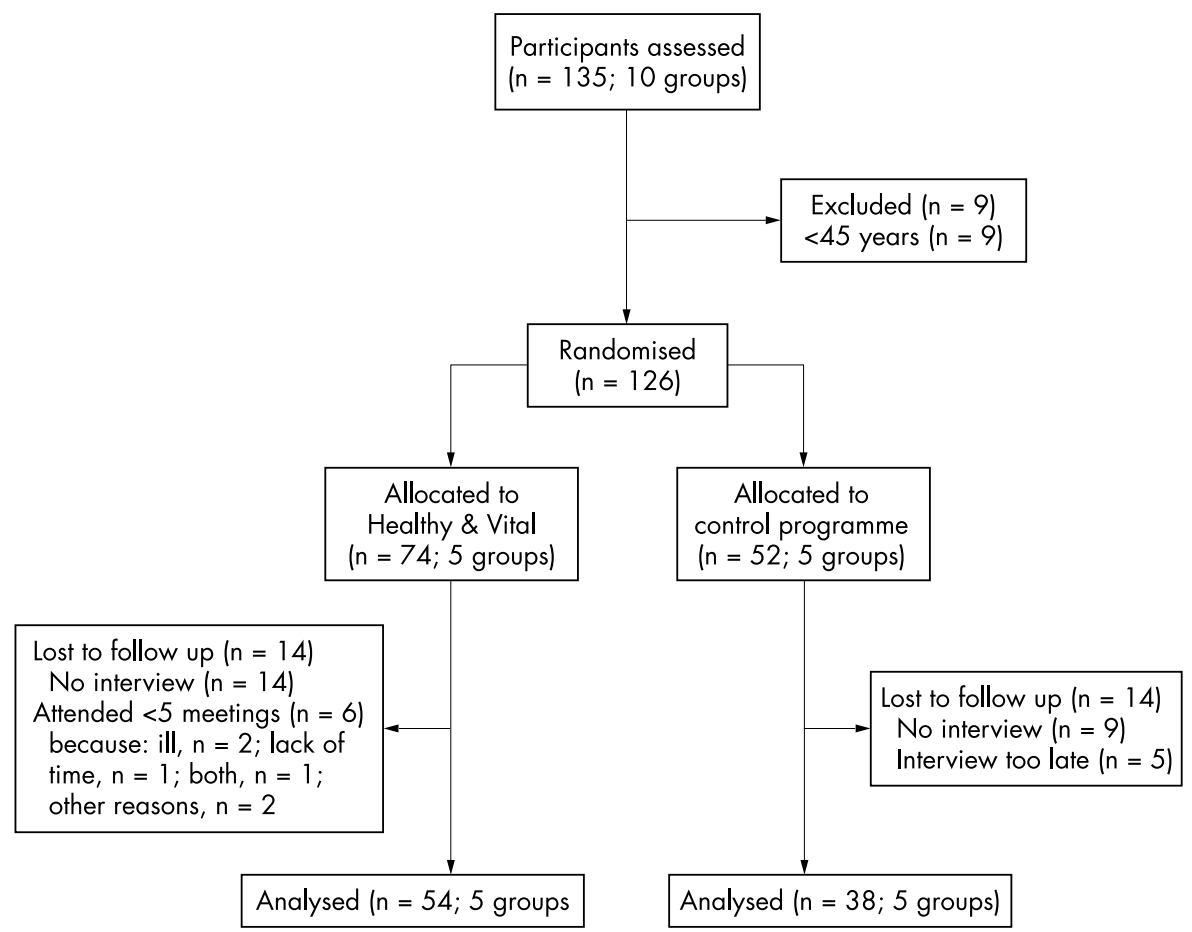

Figure 1 Participant flow and follow up.

search for better outcome measures regarding physical activity, validated and suited for Turkish elderly people, yielded no alternative. Possible ranges for all measures are shown in table 2 .

All measures were translated to Turkish by certified Turkish interpreters, and translated back by an independent native speaker to check for quality. ${ }^{24}$ SF items came from the validated Turkish version, developed by the Netherlands Cancer Institute. Finally, the interviews were piloted among Turkish elderly people who were not involved in step 3 of the adaptation of the intervention and were commented on by all Turkish interviewers during their training, leading to some modifications, especially regarding the wording of some questions and of some response categories.

\section{Data collection}

Bilingual native Turks conducted baseline interviews (within 10 days from start) and follow up interviews (eight to ten weeks later, after completion of the trial). All interviewers had been trained by the researchers and had further received written interview guidelines. During the interview periods, they were contacted at least once a week on results and on problems met. Interviewers further had to send completed questionnaires to the researchers immediately and were given instant feedback on the answers as registered (for example, on completeness and on occurrence of implausible answers).

Calculation of sample size

We designed the trial to detect a minimum important difference in effect $\operatorname{size}^{25}$ of $0.46 \mathrm{SD}$ on the outcome measures with $80 \%$ power at $\alpha=0.05$. For this, we needed 120 participants $(2 \times 60)$, using knowledge as key outcome.

\section{Analysis strategy}

We used paired $t$ tests on the differences between the Healthy \& Vital and control group regarding changes between the baseline and follow up measurements. We also computed effect sizes by dividing these differences by the standard deviations of the baseline measurements in the control group. ${ }^{25}$ People who attended less than half the sessions of a programme were excluded from these analyses as effects were unlikely in case of such a low exposure. We repeated all analyses adjusting for age and gender, and assessed effects for relevant subgroups, by dichotomising the total group on the

Table 1 Characteristics of Turkish people aged 45 and over participating in the health promotion programmes*

\begin{tabular}{lllll}
\hline & \multicolumn{2}{c}{ Analysed group } & & Lost to follow up or poor compliance \\
\cline { 2 - 4 } & $\begin{array}{l}\text { Healthy \& Vital } \\
(\mathbf{n}=54)\end{array}$ & Control $(\mathbf{n}=38)$ & $\begin{array}{l}\text { Healthy \& Vital } \\
(\mathbf{n}=20)\end{array}$ & Control $(\mathbf{n = 1 4 )}$ \\
\hline Nunber (\%) men & $12(22)$ & $11(29)$ & $2(10)$ & $3(21)$ \\
Mean (SD) age & $54.8(7.5)$ & $54.2(5.5)$ & $54.3(4.8)$ & $54.5(6.3)$ \\
Number (\%) aged 45-54 years & $28(52)$ & $20(53)$ & $10(50)$ & $7(50)$ \\
Mean (SD) number of years living in the Netherlands & $22.6(6.9)$ & $25.5(5.2)$ & $23.6(4.0)$ & $26.1(3.3)$ \\
Mean (SD) size of household & $3.2(1.5)$ & $3.5(1.8)$ & $2.5(1.2)$ & $2.4(1.1)$ \\
No (\%) not completed Turkish primary school & $28(58)$ & $20(53)$ & $13(81)$ & $8(57)$ \\
No (\%) with a Dutch diploma & $4(9)$ & $3(8)$ & $-(-)$ & $1(7)$ \\
No (\%) with considerable difficulties in speaking Dutch & $24(44)$ & $21(55)$ & $10(50)$ & $7(50)$ \\
No (\%) with at least $\geqslant 20$ min strenuous physical activity /wk & $21(39)$ & $16(42)$ & $5(25)$ & $9(64)$ \\
in summer & $18(33)$ & $15(40)$ & $5(25)$ & $7(50)$ \\
in winter & & &
\end{tabular}

*Groups differed with statistical significance regarding size of household ( $\mathrm{p}=0.025 ; F$ statistic), with smaller households in the loss to follow up groups, but not regarding any other characteristic, with $\chi^{2}$ tests or $F$ tests in analyses of variance. 


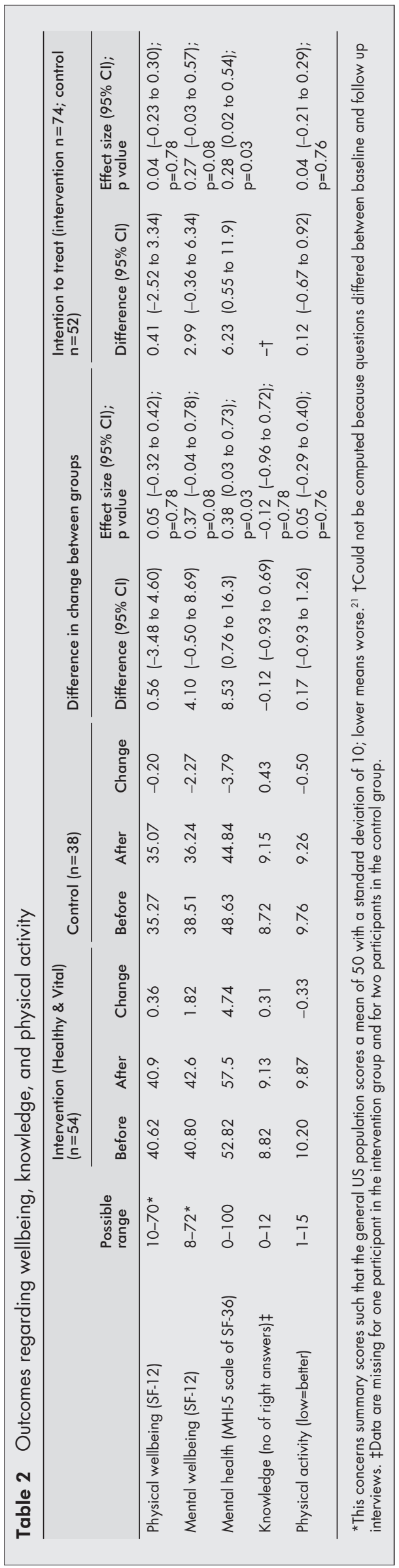

median of that characteristic: age (all outcome measures), level of physical activity (outcome: physical activity), and level of ability to speak Dutch (outcome: knowledge). Finally, we carried out an intention to treat analysis, in which we analysed data from all allocated cases, including those who attended fewer than five meetings and those lost to follow up. For the group lost to follow up, we assumed no change since baseline measurement.

\section{Assignment}

Each group of participants was allocated to the intervention or the control condition. Allocation occurred randomly in blocks of two, after stratification by gender of the group. After completion of the randomly assigned programme, all participants also received the other programme, to prevent selective loss to follow up. Therefore, the second interview had to be taken within two weeks after completion of the trial, to prevent contamination

\section{Masking}

Because of the nature of the trial, complete masking of participants and interviewers was not possible. We did not inform interviewers of the participants' trial allocation, but interviewers generally felt that, because of cultural norms regarding politeness, they could only start their interview after a general introductory conversation on mutual wellbeing, during which they sometimes got information on the programme that the participant followed. None of the interviewers had a beforehand conviction regarding the effectiveness of either the intervention or the control programme, however.

\section{RESULTS \\ Participants}

A total of 135 people entered the trial, in 10 groups, of whom 126 met the inclusion criteria. Figure 1 shows participant flow and follow up. Table 1 gives characteristics of all the people that met the inclusion criteria. Regarding physical activity, we also asked for the degree in which participants had at least 30 minutes of strenuous physical activity each day. These questions could not be filled out for over half of all participants, however, making the answers to these questions of little value (not shown). Baseline values for all outcome measures are presented in table 2 .

\section{Programme attendance and costs}

Mean attendance was 7.45 of 8 sessions among those who completed the programme (SD 0.77) $(n=54 ; 61.1 \%$ (31) attended all sessions), and 3.83 (SD 1.83) among drop outs $(n=6)$. Costs per programme were $€ 1400$; single largest contributors to costs were fees for the Turkish health educator $(€ 455)$ and the exercise instructor $(€ 240)$.

\section{Outcomes}

Table 2 gives outcomes regarding wellbeing, knowledge, and physical activity. Regarding mental health, Turkish elderly people in the Healthy \& Vital programme showed a large improvement. Although general mental wellbeing also improved, this difference was not statistically significant. Differences in change between the intervention and control group for the other outcomes were small. We found no adverse effects such as injuries because of the exercises.

Adjustment for background characteristics led to very minor changes in all outcomes (not shown). Analyses by subgroup showed an important difference in effect on mental wellbeing by age group $(p=0.04)$. Effects were larger for participants aged 55 years and over than for younger ones (table $3)$. 


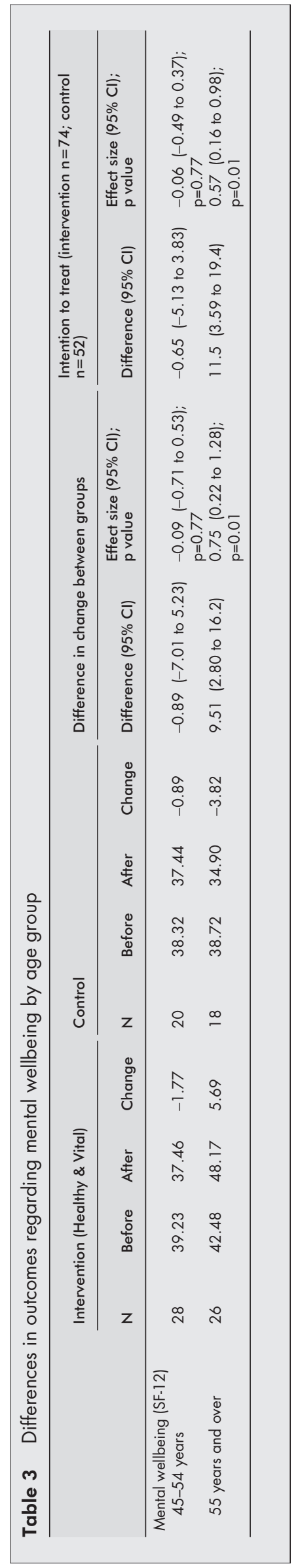

\section{Key points}

- Health problems of older immigrants from non-industrialised countries are a growing public health challenge for most Western European countries.

- These immigrants are often hard to reach through health promotion and other preventive services.

- We developed a specific programme of health education and exercises specifically for a group of elderly immigrants.

- This programme leads to an important improvement in mental health.

- Meticulous adaptations to contents and method of delivery of health promotion to this target group are essential.

\section{DISCUSSION}

The aim of this study was to assess the effect of a short health promotion programme on a group of older immigrants, aged 45 years and over, with demonstrably low incomes and low educational levels. Our results show that the programme indeed attracted people of low education, over half not having completed primary school, and that many had problems in speaking Dutch. The programme leads to an important improvement in their mental health, and in the more general mental wellbeing of those aged 55 and over. We found no effect on other outcomes such as physical wellbeing and activity or knowledge, however.

\section{Methodological considerations}

Selection bias and information bias might have influenced our findings, but such a bias is unlikely. Regarding selection bias, groups were assigned randomly and all groups received both programmes, albeit in a different sequence. This seems to have been adequate, as loss to follow up was similar in both conditions. Although the baseline characteristics of the intervention and the control group differed somewhat, these differences were mostly without statistical significance and had no impact on the size of the effects (except for age, for which both groups were very similar). Regarding information bias, we were not able to mask the condition of respondents for the interviewers. This might have affected our findings if the interviewers preferred one of the programmes. We have no indications for this, and as both programmes were specifically designed for immigrants and none of the interviewers had a prior conviction regarding the effectiveness of either of the two programmes (as we assessed during their training) we feel this is unlikely to have influenced our results.

Secondly, imprecise measurements may explain the negative findings regarding knowledge and physical wellbeing and activity. Because of follow up losses, the power of our study was lower than planned. It seems unlikely that this has affected most of our findings, as most negative findings were rather far from statistical significance. We cannot exclude the possibility that those who made quick gains dropped out early. However, to make the findings statistically significant these early quitters would have to experience unreasonably large effects. This would only be detected in an extra midprogramme interview, which could shed light on the dose-response issues within the programme but would also cause a substantial additional research burden and potentially an additional drop out. Moreover, measurement imprecision may have been comparatively large regarding some outcomes because most of them have not been validated among older Turkish respondents, but only among indigenous Dutch elderly people. Previous studies have shown a reasonable cross cultural comparability between Turks and Dutch regarding numeric outcomes such a health care utilisation, ${ }^{26}$ but this may not hold true for more complex concepts such as knowledge on health and physical activity. ${ }^{24}{ }^{27}$ We indeed found only statistically significant effects for outcomes that have been 
specifically validated among Turks (that is, regarding the SF-36/12), and not for any outcome measure for which this was not the case. Furthermore, regarding our outcome measure on physical activity, the Voorrips questionnaire, we had to exclude the items that focused on sports activities, although the effects of the programme on physical activity for indigenous elderly people concerned this part of the questionnaire. ${ }^{18}$ These modifications may have contributed to our negative findings regarding physical activity.

\section{Implications for services}

Very few studies have assessed the effectiveness of programmes aimed at promoting the health of deprived immigrants. $^{7-13}$ Our programme reached a very deprived group of immigrants and improved their mental health. This effect is highly relevant as the prevalence of mental health problems is high among immigrants. ${ }^{27-9} 28$ Our findings fit with the available evidence regarding the relation of physical exercise with psychological wellbeing in older adults, ${ }^{29}$ and regarding the positive effects of exercise on depression, ${ }^{29}{ }^{30}$ though this evidence is not fully conclusive. ${ }^{30}$

Essential for the effects of our Healthy \& Vital programme on the mental health of Turkish elderly people seems to be a painstaking adaptation of both its contents and the method of delivery to the needs of the target group. As such, besides the programme itself, the route that we followed to adapt an existing programme to older immigrants deserves wider use. In our project, this route consisted of starting with an analysis of the main health problems of the target group. We then chose a health problem to tackle-that is, lack of knowledge on health and methods to prevent health problems and lack of physical activity-based on the size of problems and on the potentials for improvement as well as on the available knowledge and skills regarding a specific topic. Next, we made a review of available programmes for that aim, regarding effectiveness in the indigenous population and estimated feasibility among the target group, leading to a preliminary choice for a specific programme- that is, Healthy \& Vital. The subsequent route consists of the steps as described: determining feasibility and key points for adaptation based on focus group interviews; adaptation of the programme based on the results of the interviews, prior experience and literature; piloting the revised programme under rigorous monitoring; and formal evaluation of the effects of the adapted programme and of problems in such a delivery at a larger scale. Finally, this route should consist of implementation at a national scale, if needed with adaptations based on the evaluation (see below).

Our study did not show effects on knowledge and physical outcomes, which may be because of its short duration and the very limited prior knowledge and physical activity of the participants. Regarding the exercise part, we think that its intensity needs to be increased. Most immigrant participants were not able to exercise at home, because of small housing and cultural barriers such as that one should not do such exercises in vicinity of younger family members, and women not in vicinity of men. The programme leads to an important improvement in their mental health, and in the more general mental wellbeing of those aged 55 and over. Regarding the entire programme, we only found effects on general mental wellbeing among those aged 55 and over, which may indicate that the widening of recruitment to include younger people, compared with the original programme that aimed at those aged 65 and over, has been too rigorous. It is further promising that almost all participants planned to enter follow up programmes (91\% regarding health education and 95\% regarding physical exercise). The next challenge is to provide such programmes so that they meet the specific cultural needs of this deprived group.

Both the provision of follow up programmes and the support of the existing Healthy \& Vital programme require a committed support organisation. Without such support, it is very difficult for hosting organisations to get things started, even though detailed manuals exist for all people involved.

\section{Implications for research}

Our results show that it is possible to conduct a controlled trial in a multi-site setting among deprived immigrant groups, although a very committed staff is needed. A methodological barrier may be the lack of measurement tools that are both cross culturally valid and sensitive to change. As such, there is still a long way to go in this area of research.

\section{ACKNOWLEDGEMENTS}

We thank all participating immigrant, healthcare, and welfare organisations for their participation. This especially cocnerns Mrs Joke Kat (Homecare Amsterdam) exercise instructor, and Mrs Tamara Baert and Polleke Verhoeven (Netherlands Institute for Health Promotion and Disease Prevention) and Tülay Beçer (Utrecht Municipal Health Service), all health educators, for their contributions to the adaptation of the programme.

\section{Authors' affiliations}

S A Reijneveld, M H Westhoff, M Hopman-Rock, TNO (Netherlands Organisation for Applied Scientific Research) Prevention and Health, Leiden, Netherlands

M H Westhoff, Netherlands Heart Foundation

M Hopman-Rock, Body@Work Research Centre on Physical activity,

Work, and Health, TNO-VU Medical Centre, Leiden, Netherlands

Funding: this study was financially supported by grant 9607.021 .3 from the Dutch Health Research and Development Council (Zon Mw).

Conflicts of interest: none.

\section{REFERENCES}

1 Carballo M, Divino JJ, Zeric D. Migration and health in the European Union. Trop Med Int Health 1998:3:936-44.

2 Reijneveld SA. Reported health, lifestyles, and use of health care of first generation immigrants in The Netherlands: do socioeconomic factors explain their adverse position? J Epidemiol Community Health 1998;52:298-304.

3 Lewinter M, Kesmez SS, Gezgin K. Self-reported health and function status of elderly Turkish immigrants in Copenhagen, Denmark. Scand J Soc Med 1993;21:159-63.

4 Bayard-Burfield L, Sundquist J, Johansson SE. Self-reported long-standing psychiatric illness and intake of benzodiazepines. Eur J Public Health 2000;10:51-7.

5 Pudaric S, Sundquist J, Johansson SE. Impaired mobility and impaired working capacity among foreign born people and native born Swedes. J Epidemiol Community Health 1998;52:34-40.

6 Sundquist J. Ethnicity, social class and health. A population-based study on the influence of social factors on self-reported illness in 223 Latin American refugees, 333 Finnish and 126 south European labour migrants and 841 Swedish controls. Soc Sci Med 1995;40:777-87.

7 Ebrahim S. Ethnic elders. BN 1996;313:610-13.

8 Silveira ER, Ebrahim S. Social determinants of psychiatric morbidity and well-being in immigrant elders and whites in east London. Int J Geriatr Psychiatry 1998;13:801-12

9 Rait G, Burns A. Appreciating background and culture: the South Asian elderly and mental health. Int J Geriatr Psychiatry 1997;12:973-7.

10 Sundquist J. Migration, equality and access to health care services. $J$ Epidemiol Community Health 2001:55:691-2.

11 Arblaster L, Lambert M, Entwistle V, et al. A systematic review of the effectiveness of health service interventions aimed at reducing inequalities in health. J Health Serv Res Policy 1996;1:93-103.

12 Taylor WC, Baranowski T, Young DR. Physical activity interventions in low-income, ethnic minority, and populations with disability. Am J Prev Med 1998;15:334-43.

13 Krumeich A, Weijts W, Reddy P, et al. The benefits of anthropological approaches for health promotion research and practice. Health Educ Res 2001;16:121-30.

14 Stronks K, Ravelli AC, Reijneveld SA. Immigrants in the Netherlands: equal access for equal needs? J Epidemiol Community Health 2001;55:701-7

15 Poort EC, Spijker J, Dijkshoorn H, et al. Turkish and Moroccan elderly in Amsterdam, 1999-2000. [In Dutch]. Amsterdam: Municipal Health Service, 2001.

16 Dijkshoorn H. Unhealthy lifestyles in Amsterdam: differences between Turks, Moroccans and Dutch. [In Dutch]. Amsterdam: Municipal Health Services, 2001

17 Hopman-Rock $M$, Westhoff $M H$. Health education and exercise stimulation for older people: development and evaluation of "Healthy \& Vital". [In Dutch]. Tiidschr Gerontol Geriatr 2002;33:56-63. 
18 Hopman-Rock M, Westhoff MH. Development and evaluation of "Aging Well and Healthily": a health education and exercise program for community-living older adults. Journal of Aging and Physical Activity 2002; 10:363-80.

19 Hickey T, Wolf FM, Robins LS, et al. The SMILE Program. Ann Arbor: University of Michigan, School of Public Health, 1992

20 Reijneveld SA, Van Rooij EHC, Westhoff MH. The feasibility of the programme "Healthy \& Vital" for Turkish and Moroccan elderly. [In Dutch]. Leiden: TNO Prevention and Health, 1998.

21 Ware JE, Kosinski M, Keller SD. SF-12: how to score the SF-12 physical and mental health summary scales. Boston, MA: New England Medical Center, the Health Institute, 1995.

22 Gandek B, Ware JE, Aaronson NK, et al. Cross-validation of item selection and scoring for the SF-12 Health Survey in nine countries: results from the IQOLA Project. International Quality of Life Assessment. $J$ Clin Epidemiol 1998:51:1171-8.

23 Voorrips LE, Ravelli AC, Dongelmans PC, et al. A physical activity questionnaire for the elderly. Med Sci Sports Exerc 1991;23:974-9.

24 Guillemin F, Bombardier C, Beaton D. Cross-cultural adaptation of health-related quality of life measures: literature review and proposed guidelines. J Clin Epidemiol 1993;46:1417-32.
25 Cohen J. Statistical power analysis for the behavioral sciences. Hillsdale: Lawrence Erlbaum, 1988.

26 Reijneveld SA. The cross-cultural validity of self-reported use of health care: a comparison of survey and registration data. J Clin Epidemiol 2000;53:267-72

27 Johnson T, O'Rourke D, Chavez N, et al. Social cognition and responses to survey questions among culturally diverse populations. In: Lyberg L, Biemer P, Collins C, et al, eds. Survey measurement and process quality. New York: Wiley, 1997:87-113.

28 Gonzalez HM, Haan MN, Hinton L. Acculturation and the prevalence of depression in older Mexican Americans: baseline results of the Sacramento Area Latino Study on Aging. J Am Geriatr Soc 2001;49:948-53.

29 Biddle S, Faulkner, G. Psychological and social benefits of physical activity. In: Chan KM, ed. Active aging. Hong Kong: Lippincott, 2002:89-164.

30 Lawlor DA, Hopker SW. The effectiveness of exercise as an intervention in the management of depression: systematic review and meta-regression analysis of randomised controlled trials. BM 2001;322:763-7.

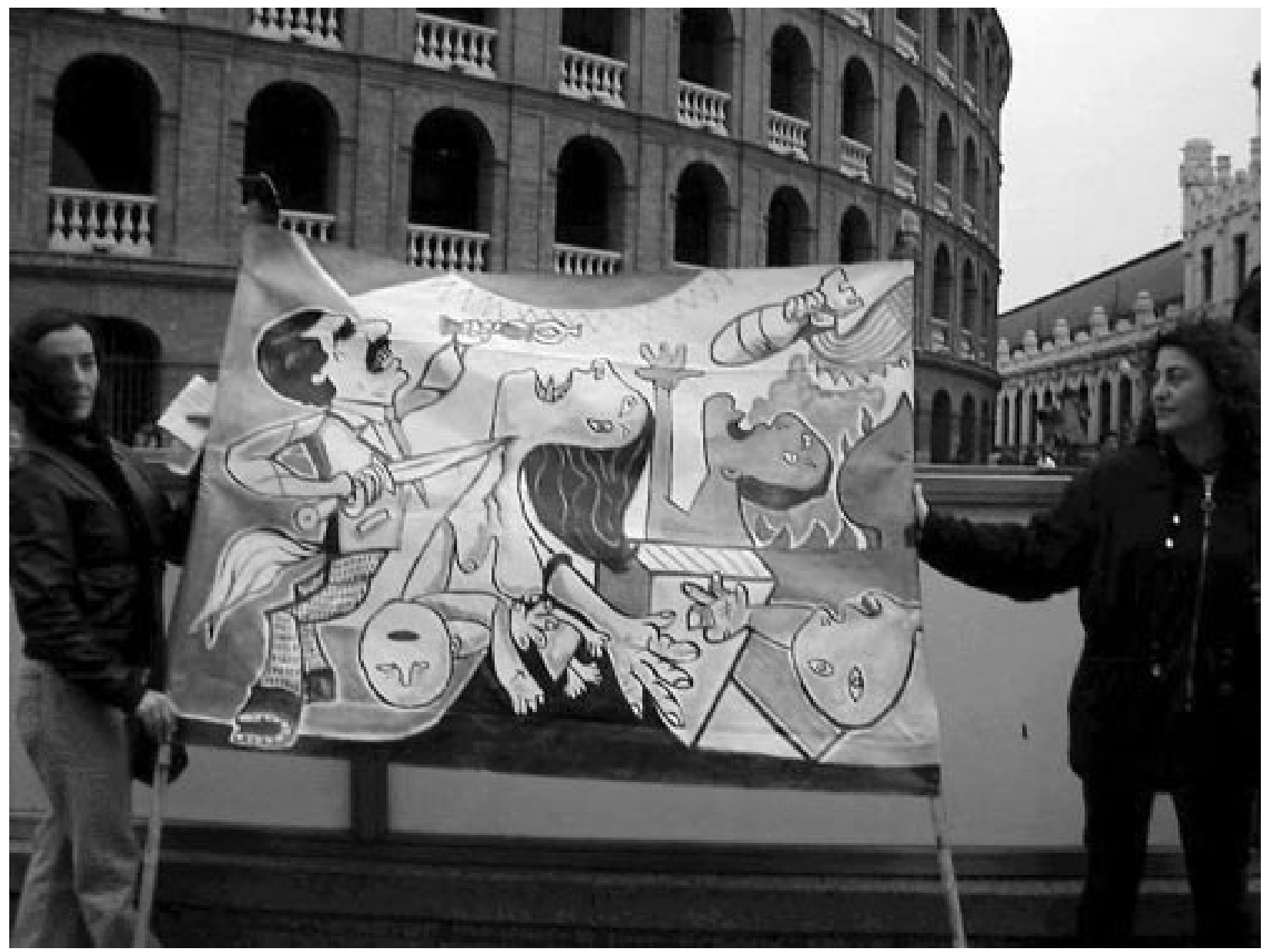

Guernica revisited in Valencia, Spain, 2003. 